\title{
Temas y motivaciones de niñas, niños y adolescentes al crear humor gráfico
}

Lucía Bugallo, Lic.

ECyC IPEHCS Conicet, Universidad Nacional de Comahue, Argentina.*

Ana Pedrazzini, PhD.

Conicet, Argentina**

\author{
Constanza Zinkgräf, Mg. \\ ECyC IPEHCS Conicet, \\ Universidad Nacional de Comahue, Argentina.*** \\ Nora Scheuer, PhD. \\ ECyC IPEHCS Conicet, \\ Universidad Nacional de Comahue, Argentina. ${ }^{\star \star \star \star}$
}

\section{Resumen (analítico)}

El humor gráfico es un medio que niños, niñas y adolescentes encuentran atractivo para dar cuenta de vivencias personales y presentar su perspectiva ante un tema que los convoca. Son escasos los estudios que han abordado de forma articulada aspectos pragmáticos motivacionales y temáticos en el humor gráfico de adolescentes. Con este propósito, realizamos un análisis multidimensional de 151 textos multimodales realizados por niños, niñas y adolescentes de 10 a 19 años en nueve talleres implementados en contextos de educación formal e informal en Argentina. A partir de análisis multivariados identificamos diferencias etarias y de formato (viñeta única o tira) según la motivación y los temas de los textos.

\section{Palabras clave}

Psicología del desarrollo, infancia, adolescencia, artes visuales, motivación, humor.

\section{Thesauro}

Tesauro de Ciencias Sociales de la Unesco.

\section{Para citar este artículo}

Bugallo, L., Pedrazzini, A., Zinkgräf, C., \& Scheuer, N. (2020). Temas y motivaciones de niñas, niños y adolescentes al crear humor gráfico. Revista Latinoamericana de Ciencias Sociales, Niñez y Juventud, 18(2), 1-28. http://dx.doi.org/ $10.11600 / 1692715 \times .18210$

\section{Historial}

Recibido: 07.10.2019

Aceptado: 20.02 .2020

Publicado: 25.05.2020

Información artículo

Artículo resultado de los proyectos de investigación «Aprendizaje y comunicación como procesos multimodales en aprendices y profesionales», financiado por la Universidad Nacional del Comahue (C107, 01/01/2017-en curso) y «Un enfoque múltiple, dinámico y contextual de la alfabetización: Aprendizaje y uso de diversos sistemas semióticos», financiado por el Consejo Nacional de Investigaciones Científicas y Técnicas (PIP 2014-0142, 21/09/2016-en curso). Área: Ciencias Sociales; subárea: Ciencias Sociales, Interdisciplinaria. 


\section{Topics and motivations of children and adolescents when creating comics and cartoons}

\section{Abstract (analytical)}

Cartooning is a medium that is used by young people to share personal experiences and express their perspective on a topic that they find appealing. Few studies have addressed the motivational, pragmatic and thematic aspects of adolescents' cartoons in an articulated manner. With this purpose, we carried out a multidimensional analysis of 151 multimedia texts created by children and adolescents aged 10 to 19 years old during 9 workshops that were implemented in formal and informal education contexts in Argentina. Multivariate analyses allowed us to identify age-based and format differences (single panel or comic strips) based on the children and adolescents' motivation and themes.

Keywords

Developmental psychology, childhood, adolescence, visual arts, motivation, humor.

\section{Temas e motivações de crianças e adolescentes na criação de humor gráfico}

Resumo (analítico)

O humor gráfico é um meio através do qual crianças e adolescentes acham atrativo explicar experiências pessoais e apresentar suas perspectivas diante de um tópico que os reúne. Existem poucos estudos que abordaram de forma articulada os aspectos pragmáticos motivacionais e temáticos no humor gráfico dos adolescentes. Com esse propósito, realizamos uma análise multidimensional de 151 textos multimodais feitos por crianças e adolescentes de 10 a 19 anos em nove oficinas implementadas em contextos de educação formal e informal na Argentina. A partir da análise multivariada, identificamos diferenças de idade e formato (vinheta única ou tira) de acordo com a motivação e os temas dos textos.

\section{Palavras-chave}

Psicologia do desenvolvimento, infância, adolescência, artes visuais, motivação, humor.

\section{Información autoras}

[*] Becaria doctoral del Consejo Nacional de Investigaciones Científicas y Técnicas, en el grupo vinculado de Estudios Culturales y Cognitivos, Instituto Patagónico en Humanidades y Ciencias Sociales (Ipehcs), Conicet, Universidad Nacional del Comahue, Argentina. Licenciada en Psicología, Universidad del Aconcagua. Índice H5: 4. Correo electrónico: luciabugallo@gmail.com iD 0000-0003-3241-338X.

[**] Investigadora del Consejo Nacional de Investigaciones Científicas y Técnicas, en el grupo vinculado de Estudios Culturales y Cognitivos, Ipehcs, Conicet, Universidad Nacional del Comahue, Argentina. Doctora en Ciencias de la Información y la Comunicación, Universidad Paris-Sorbonne y Doctora en Ciencias Sociales, Universidad de Buenos Aires. iD 0000-0003-0524-0305. Índice H5: 5. Correo electrónico:

ana.pedrazzini@crub.uncoma.edu.ar

[***] Profesora e Investigadora de la Universidad Nacional del Comahue. Asistente de docencia regular. Licenciada en Psicología, Universidad de Buenos Aires y Master en Educación, Universidad de Jaén, España. Índice H5: 1. Correo electrónico: c.zinkgraf@gmail.com (iD) 0000-0003-0926-2106.

[****] Investigadora del Consejo Nacional de Investigaciones Científicas y Técnicas, en Ipehcs, Conicet, Universidad Nacional del Comahue, Argentina. Profesora Universidad Nacional de La Plata. Psicopedagoga, Universidad Caece. Doctora en Psicología, Université de Genève. Índice H5: 14. Correo electrónico: nora.scheuer@gmail.com (iD) 0000-0002-3273-9323. 


\section{Introducción}

$\mathrm{C}_{\text {gan una serie de desafíos comunicacionales para su autor: lograr una buena re- }}$ humor gráfico es un tipo particular de producción gráfica, en la cual se conjupresentación (mímesis), «hacer entender» algo a un otro ausente (Steimberg, 2013) y, además, crear un efecto humorístico que suscite sorpresa ante la incongruencia percibida, seguida por una amplia gama de potenciales reacciones que van desde la diversión y el disfrute hasta la conmoción o el disgusto.

El humor gráfico conforma un género discursivo (Pedrazzini \& Scheuer, 2018), reconocido en textos en sentido amplio (Vilches, 1984 ) exclusivamente visuales o multimodales (Kress, 2010), que por lo general articulan los modos visual y verbal escrito. Suele desplegarse en un espacio reducido (una viñeta única o una tira de pocos cuadros) en el cual se plasma sintéticamente el clímax humorístico (Pitri, 2011) de la situación representada, dando lugar a una trama autoconclusiva.

Entendemos la creación de humor gráfico como un «juego narrativo gráfico» (Wright, 2007), en el cual el creador asume diversos roles: autor, director, guionista, narrador, artista. En este sentido, crear humor gráfico exige y promueve un conjunto de desafíos en diversos planos que revisten interés para el ámbito educativo. Además de los ya señalados, destacamos su gran potencial como un medio que permite a niños, niñas y jóvenes dar cuenta de vivencias personales, jugar con el orden establecido y presentar su punto de vista ante un tema que los convoca (Bugallo et al., 2018a).

Si bien diversos estudios han abordado la interpretación de humor gráfico por parte de niños y adolescentes (Puche-Navarro \& Lozano-Hormaza, 2002), se conoce poco acerca de los temas que ellos mismos abordan (entre las excepciones, Piret, 1940; Pitri, 2011) y los motivos que los impulsan al crear humor gráfico por sí mismos. Este trabajo busca aportar en esa dirección, mediante un abordaje multidimensional e integrativo de los temas y las motivaciones de niños, niñas y adolescentes al crear humor gráfico. 
Antes de adentrarnos en nuestro estudio, los próximos apartados estarán abocados, en primer lugar, a presentar algunas características constitutivas de este género discursivo, para luego presentar algunos antecedentes encontrados sobre los aspectos pragmáticos motivacionales en producciones gráficas de niños, niñas y adolescentes. Seguidamente, mencionaremos ciertas características relacionadas con el desarrollo del humor, focalizando en aspectos temáticos. Así mismo, analizaremos diversas clasificaciones temáticas realizadas sobre producciones gráficas de ellos y ellas.

\section{Algunas características del humor gráfico como género discursivo}

El humor gráfico tuvo su origen en la prensa, con un fuerte énfasis en la crítica política. Con el paso del tiempo, este medio de comunicación se fue diversificando en cuanto a temas y motivaciones de los autores, dando lugar a distintos subgéneros (Pedrazzini \& Scheuer, 2018) que circulan a través de múltiples canales.

Como vimos, una condición básica de este género es la creación de un efecto humorístico. Siguiendo la teoría de la incongruencia (Attardo, 1997), el humor tiene lugar cuando se introduce un desajuste, una situación que viola nuestras expectativas produciendo así una sorpresa, que el lector o destinatario disfruta únicamente si se predispone al juego de «como si», a través de un modo mental lúdico o paratélico (Apter, 1991). Predisponerse a este juego habilita el despliegue de múltiples sentidos, incluso el sinsentido (Charaudeau, 2006). En su acepción contemporánea más extendida, el humor se asocia con la creación y percepción de estímulos divertidos que tienden a hacer reír a otros (Martin \& Ford, 2018). Sin embargo, diversos enfoques permiten contemplar otro tipo de motivación en la producción humorística. Cuando lo dominante es lo reflexivo, se genera una distancia sobre la percepción de lo incongruente. Es el caso del «humor serio» (Flores, 2000) en el cual subyace una motivación comprometida por parte del autor, cuyo propósito es denunciar y alertar sobre una situación que le produce indignación, ira o dolor (Freud, 1927/1992; Pedrazzini \& Scheuer, 2010), si bien puede coexistir con una motivación lúdica (Pedrazzini \& Scheuer, 2018). En este caso, el disfrute puede basarse en la interpretación del sentido, que implica encriptar y desencriptar un mensaje, que no necesariamente es risible (Bugallo et al., 2018b).

Otro aspecto relevante para entender el funcionamiento del humor gráfico refiere al tratamiento de una situación del mundo real o bien inspirada en este (a la que llamaremos, siguiendo a Pedrazzini \& Scheuer, 2010, 2018, situación referida) mediante la repre- 
sentación de una situación ficticia (Pedrazzini \& Scheuer, 2010, 2018). Como veremos más adelante, la representación ficcional puede ser realista, en tanto se mantiene dentro del orden de lo posible y verosímil (Attardo \& Raskin, 1991), o bien fantástica, al subvertir el orden posible y crear una situación inverosímil.

En el humor gráfico confluyen diversos recursos multimodales específicos del género, como globos de diálogo, cartuchos, onomatopeyas y etiquetas, que se articulan en la producción de sentido. Además, el humor gráfico se puede presentar en dos formatos: tira o viñeta única. La tira ofrece un espacio en el cual se despliegan acciones y, a su vez, genera un efecto de temporalidad en la narrativa (McCloud, 1994). Muchas veces la incongruencia que se presenta en este formato es de tipo mecánica, dado que son los elementos presentes en cada viñeta los que contrastan con el final inesperado en la viñeta final (Puche-Navarro \& Lozano-Hormaza, 2002). Por su parte, la viñeta única conforma un texto autocontenido que generalmente no admite expansiones, sino que propone un hiato, requiriendo del lector la simultánea comprensión de los sentidos propios y figurados puestos en juego. En un estudio previo con adolescentes, encontramos una asociación entre la presencia de incongruencias mecánicas como las equivocaciones o «meteduras de pata» y el formato en tiras, mientras que la viñeta única se asoció con incongruencias más sofisticadas de tipo conceptual (Pedrazzini et al., en prensa).

\section{La dimensión pragmático-motivacional en las} creaciones gráficas de niñas, niños y adolescentes

Si bien poco se conoce acerca de las motivaciones de niños, niñas y adolescentes al crear humor gráfico, la abundante investigación acerca de las decisiones que toman cuando realizan dibujos figurativos o bien narrativas no específicamente humorísticas, así como sus intereses y motivaciones, nos brindan algunas pistas de interés.

Los estudios en el campo del dibujo revelan que los niños ajustan y organizan la información que plasman en el papel guiados tanto por intenciones comunicativas como por juicios de relevancia de orden conceptual (Bombi \& Pinto, 1993; Freeman, 1980). Esto explica la tendencia de muchos niños a realizar dibujos canónicos de los objetos que buscan graficar, a fin de asegurar que sean fácilmente reconocibles por los lectores (Pitri, 2011). De forma similar, el recurso al dibujo esquemático no necesariamente implica escasa maestría gráfica, sino que puede orientarse a favorecer la fluidez en la lectura, guiando la atención del lector sobre los eventos narrados y no sobre aspectos plásticos. Progresivamente, los aspectos expresivos y formales son ajustados también según la 
audiencia prevista (Burkitt et al., 2011), aunque durante la mediana infancia en ocasiones acuden al modo oral para explicitar aspectos visuales (Labitsi, 20o8; Pitri, 2011).

Por otra parte, las motivaciones de los autores están en relación con sus intereses y preocupaciones, que cambian en el tiempo y se vinculan a la experiencia de vida. Por ello se ha propuesto que, en las producciones gráficas, los signos portan una doble motivación social: una dada por el contexto social de producción, que conlleva una evaluación del ambiente comunicacional en el cual se ponen en marcha determinados ajustes en y para la creación textual (Kress, 2010), y otra dada por quién es y cuál ha sido la historia de quien crea los textos. En cuanto a la primera vía de motivación social, numerosos estudios han demostrado que las personas copresentes y sus comentarios impactan en el proceso del dibujo, así como en los significados que se construyen (Einarsdottir et al., 2009). En cuanto a la segunda vía, un ejemplo de cómo la experiencia personal impacta en la creación textual es el de un estudio a través de talleres creativos orientados a la educación para la paz de niños y niñas de entre siete y doce años de edad. Este estudio de caso reveló que el contenido de los dibujos creados por estos niños se relacionaba con el grado en que las zonas en las que ellos vivían habían sido afectadas por conflictos armados (Ospina-Ramírez et al., 2018). En conjunto, todos estos estudios dan cuenta de una profunda imbricación entre los dibujos realizados y los contextos próximos y más amplios de producción.

\section{La dimensión temática en las creaciones humorísticas}

\section{de niñas, niños y adolescentes}

Entendiendo al humor como una actividad intelectual deliberada, íntimamente ligada al desarrollo cognitivo, comunicativo y social (McGhee, 1979; Milreault \& Reddy, 2016), los motivos, tipos y temas que conforman el repertorio humorístico de niños y adolescentes varían durante su desarrollo. Desde la mediana infancia, y a lo largo de toda la adolescencia, el humor juega un importante rol a escala interpersonal como factor de cohesión grupal entre pares (Martin \& Ford, 2018). La burla también es muy frecuente en la interacción de pares y gira en torno a la apariencia física, equivocaciones o «meteduras de pata», situaciones escatológicas o el desempeño intelectual y físico (Bariaud, 2013).

Durante la adolescencia temprana, si bien persiste un gran interés por chistes conocidos, adivinanzas y colmos (McGhee, 2002), se registra un notable cambio en el tipo de humor: cobran relevancia aquellas formas que exigen resolver incongruencias para crear sentido cognitivo (Bariaud, 2013). Los valores e intereses devienen objetos de reflexión (Rew et al., 2012) y algunos adolescentes se interesan profundamente por fenómenos socia- 
les (Karakos, 2015; Moshman, 2009), que dan lugar a la burla y a la crítica a escala social (Bariaud, 2013). El desarrollo del perspectivismo y de los procesos metacognitivos permite la exploración de múltiples posibilidades de interpretación y creación de juegos de sentidos sobre diversas temáticas. De la mano de una progresiva capacidad para utilizar el repertorio convencional de expresiones figurativas (Levorato \& Cacciari, 2002), los adolescentes prefieren manifestaciones de humor más sofisticadas, basadas en contenidos abstractos y que involucran retos cognitivos mayores (Martin \& Ford, 2018; McGhee, 1979). Esto va de la mano con la progresiva consideración del efecto que un texto tiene sobre sus destinatarios o lectores potenciales (Hess-Zimmermann, 2014). Así, el humor se torna más espontáneo y original (Klein \& Kuiper, 2006). Entre los tópicos humorísticos, abundan las ocurrencias o anécdotas que enfatizan conflictos sociales, temas tabú y contenido sexual (Bariaud, 2013).

Además de las escalas social e interpersonal, el humor de los adolescentes también puede tratar sobre sus propias experiencias de vida, ya sea como estrategia de afrontamiento ante dificultades que encuentran o, por el contrario, como una vía para evitarlas (Führ, 2002).

\section{Criterios de clasificación temática en producciones gráficas}

La revisión de la literatura sobre producciones gráficas revela una gran diversidad de criterios para abordar la dimensión temática. Los trabajos centrados en producciones específicamente humorísticas se caracterizan, por un lado, por una concepción del humor restringida al carácter lúdico y, por otro, por un abordaje dicotómico. Es el caso de los estudios pioneros de Piret (1940) y Pitri (2011) sobre dibujos realizados por niños y niñas de 4 a 12 años. Una categoría refiere a «características cómicas» (Piret) o «personajes» (Pitri), y consiste en la representación de figuras u objetos en contextos neutrales o no significativos (característico de los niños menores). Pitri encontró que los niños generalmente dibujan figuras consideradas canónicamente como divertidas (por ejemplo, payasos), criaturas imaginarias y personajes de dibujos animados. La otra categoría ha sido llamada «situaciones y comportamientos cómicos» (Piret) o «incidentes» (Pitri), que consiste en la representación de personajes desplegando acciones (característico de los niños mayores).

Encontramos cierta resonancia entre ambas categorías y el árbol ontológico propuesto por Chi (1992, 2008), que busca captar a través de categorías taxonómicas ciertas existencias en el mundo, tal como se han abordado desde la filosofía. En este sentido, los 
«personajes» corresponderían a «materia» y los «incidentes» a «eventos». La tercera ontología propuesta por Chi, «abstracciones», permitiría complejizar las entidades que suelen ser representadas en el humor gráfico.

Con el fin de rastrear categorías temáticas más detalladas, ampliamos la búsqueda para incluir dibujos no necesariamente humorísticos. En los casos de temática libre sin restricción de aspectos expresivos, se ha encontrado que el entorno inmediato, las personas importantes para los niños, así como diversos dispositivos (cuentos, videos, juegos de computadora, etc.) forman parte de los temas de sus dibujos (Faulkner \& Coates, 2011).

Quizás la categorización temática más exhaustiva sea la de Wilson y Wilson (1987) sobre narrativas gráficas realizadas por niños y niñas de 9 a 12 años. Establecieron trece categorías: procesos naturales, creación/construcción, vida cotidiana, odisea/aventura, amistad/vínculos, asistencia, entrega/regalos, éxito/recompensa, fracaso/derrotas, peleas/ competencias/guerra, desafíos, muerte/destrucción e infortunio/accidentes. Esta clasificación, a nuestro entender, no escapa a cierta superposición; tal es el caso de las peleas/ competencia/guerra y muerte/destrucción.

Fundamentados en estos antecedentes, proponemos entender la construcción temática como multidimensional, en la cual el autor opta por fuentes del mundo real, mediático o imaginario, así como por representar esos mundos de forma realista o fantástica; entidades concretas, como personajes o eventos, o bien abstractas; escalas personales, interpersonales o sociales; y contenidos relacionados con la agresión, el amor y la amistad, los juegos, las equivocaciones, los infortunios, entre otros.

Este estudio propone un análisis articulado de aspectos pragmáticos motivacionales y temáticos en la creación de humor gráfico de niños y adolescentes, para responder a las siguientes preguntas de investigación: ¿cuál es la motivación de los autores al crear humor gráfico?, ¿existe una preferencia por fuentes reales, imaginarias o mediáticas?, ¿qué tipo de entidades dibujan?, ¿las situaciones ficticias construidas son de carácter realista o fantástico?, ¿qué contenidos abordan los adolescentes y a qué escala?, ¿varían tanto los temas abordados como el tipo de motivación en función de la edad de los participantes?, ¿incide el formato del texto en los temas y motivaciones elegidos?

A modo de hipótesis, y basándonos en los antecedentes, esperamos una preferencia por textos con motivación lúdica, así como contenidos de burla y equivocaciones en los participantes de 10 a 12 años; y un progresivo pasaje a un humor más comprometido en los años subsiguientes, con un creciente abordaje de temas sobre problemáticas sociales 
y temas tabú especialmente en los adolescentes mayores. Así mismo, es de esperar que la inclusión de entidades abstractas en los dibujos ocurra también en los participantes de mayor edad. Además, es probable que las condiciones sociales de producción (talleres de producción humorística en contextos educativos, sean formales o informales) incidan en la elección de los contenidos abordados, limitando la expresión de temáticas escatológicas y tabú. En cuanto al formato textual, se espera que, aprovechando el despliegue espacial secuencial de las tiras, estas se utilicen preferentemente para representar acontecimientos, mientas que las viñetas únicas se asocien con contenidos sociopolíticos, formato privilegiado en la prensa.

\section{Método}

\section{Procedimientos e instrumentos}

Entre 2015 y 2018 tres de las autoras realizamos diez talleres de producción e interpretación de humor gráfico en contextos educativos formales e informales (tres escuelas públicas, una escuela privada, un Centro Municipal de Desarrollo de la Niñez y Adolescencia, un Centro Integrador Comunitario y un Encuentro Nacional de Clubes de Ciencia, organizado por el Ministerio de Ciencia y Tecnología de la Nación), en diversos barrios de las ciudades de San Carlos de Bariloche, General Roca y Dina Huapi en Argentina. El primer taller, de carácter exploratorio, nos permitió conocer el interés de los adolescentes por crear humor gráfico, así como indagar las estrategias utilizadas en su creación. En este caso no se conversó previamente con los participantes sobre el género ni se presentó material. Identificamos dos estrategias en la producción humorística: 55 \% plasmaron gráficamente un chiste conocido, mientras que el $41 \%$ restante representó equivocaciones.

Con base en la información registrada (el interés suscitado por la consigna, así como las estrategias para su realización), diseñamos los restantes talleres con el objetivo de brindar más elementos acerca del género. Estos consistieron en un único encuentro, dividido en tres etapas. Tras un intercambio inicial sobre qué es el humor gráfico, se proyectó una presentación especialmente diseñada para el taller, durante la cual se alentó la participación en un clima distendido. Los contenidos fueron presentados de forma progresiva e integrada mediante viñetas humorísticas. Se abordó una variedad de temas:

- Autores y estilos (autores profesionales de distintos países y autores niños, niñas y adolescentes participantes de talleres previos). 
- Formatos (viñetas únicas y tiras).

- Temas: desde temas lúdicos (equivocaciones, subversión del orden establecido, etc.), hasta temáticas ubicadas bajo el término «humor serio» (Flores, 2000) como discriminación, violencia de género, contaminación ambiental, etc.

- Recursos retóricos: hipérbole, personificación, metáfora, paradoja, alusión, ironía, metonimia, antítesis, final inesperado, ironía del destino, etc.

- Recursos plásticos: producciones en blanco y negro, en color, en lápiz, en tinta, con diferentes tipografías, ángulos de toma, planos, etc.

- Recursos específicos del género: onomatopeyas, globos, ideogramas, líneas cinéticas, cartuchos, carteles, etiquetas, títulos y finales.

Esta actividad tuvo una duración aproximada de zo minutos. Finalmente, los participantes crearon individualmente un texto humorístico, de libre elección temática y formato, para lo cual contaron con diversos materiales gráficos (hojas, lápices de colores, fibras, reglas, libros de dibujo). La mayoría de los participantes intervinieron con entusiasmo, compromiso y evocando sus propias experiencias. El proceso de producción tuvo una duración máxima de 50 minutos. Algunos participantes encontraron dificultades en la elección de la temática y, en ocasiones, en el modo de plasmarla gráficamente. Las investigadoras colaboraron recordando el carácter exploratorio de la actividad. Cada participante fue luego entrevistado acerca de su producción y del origen de la misma. Cada entrevista duró entre tres y cinco minutos.

\section{Participantes y corpus}

En los nueve talleres que son objeto de esta investigación participaron 132 adolescentes de 10 a 19 años (62 mujeres y 70 varones). El criterio de inclusión de los participantes fue su voluntad para desarrollar la actividad y la firma de un consentimiento informado por sus padres, tutores o, en algunos casos, por los directivos de las instituciones. El corpus o conjunto de textos humorísticos que conforma el objeto de análisis de esta investigación es de 151. Cabe aclarar que algunos participantes realizaron más de una producción.

\section{Dimensiones y categorías de análisis}

Construimos categorías motivacionales y temáticas articulando aportes teóricos previos con un abordaje inductivo del corpus inspirado en la teoría fundamentada (Glaser \& Strauss, 1967 ). 
La dimensión pragmático-motivacional comprende las siguientes categorías mutuamente excluyentes, provenientes de Pedrazzini y Scheuer (2018):

- Motivación comprometida: el propósito del autor es denunciar, alertar o conmover al lector respecto de una situación que se considera indignante, dolorosa, peligrosa, etc.

- Motivación lúdica: el objetivo del autor es divertir o entretener al lector.

- Motivación mixta: se propone el abordaje de una temática que compromete, conmueve o impacta al lector, conservando el tono lúdico que amortigua el efecto.

La dimensión temática comprende cuatro subdimensiones: orden, ontología, escala y contenidos. Las categorías de las primeras tres subdimensiones son mutuamente excluyentes, de modo que cada producción remite a un único orden, ontología y escala. En cambio, dado que una misma producción suele poner en juego más de un contenido, en esa subdimensión se codifican todas las categorías identificadas.

\section{Mundo-representación}

Inicialmente distinguimos la situación referida y la situación ficticia. A continuación determinamos si la situación referida correspondía a fenómenos que pueden ocurrir en el mundo real, a creaciones que provienen en cierto grado de la imaginación del autor o bien remiten a juegos intertextuales provenientes de elementos culturales conocidos que circulan en la sociedad, como cuentos, personajes de series, películas, chistes, expresiones figuradas. En segunda instancia determinamos si la situación ficticia se inscribía también en el mundo real, en el cual los personajes realizan acciones posibles, poniendo en juego así un tratamiento realista; o bien desplegaba una construcción representacional fantástica. El carácter fantástico implica la subversión del orden existente y la violación de las reglas físicas y naturales, resultando en una representación inverosímil por los elementos irreales o combinaciones imposibles de elementos reales que la componían (Attardo \& Raskin, 1991). Las categorías resultantes son las siguientes:

- Real-realista: la situación referida trata de algo que ocurrió o puede ocurrir y se representa de manera realista. El texto adquiere así un efecto de verosimilitud.

- Real-fantástico: mientras que la situación referida es propia del mundo real, la ficticia adquiere un tono fantástico. 
- Imaginario/mediático-fantástico: incluye tanto contenidos de amplia circulación social como contenidos imposibles inventados por el autor, que construyen una situación ficticia de carácter fantástico.

\section{Escala}

Alcance del tema, del más restringido al más amplio.

- Personal: se focaliza en la esfera interna o en experiencias de primera persona, ya sea del personaje o del autor.

- Interpersonal: involucra a dos o más personajes y el foco está puesto en su interacción.

- Social: involucra a una comunidad o a toda la humanidad. Cabe mencionar que en los pocos casos en los que la producción podía ser codificada tanto como interpersonal como social, optamos por la más abarcativa, es decir, la escala social.

\section{Ontología}

Adaptamos las tres categorías básicas del árbol ontológico propuesto por Chi (1992) para dar cuenta de la naturaleza ontológica de las principales entidades plasmadas en el texto.

- Personaje: la trama se basa únicamente en el dibujo de un personaje (humano, animal u objeto personificado) e incluye lo que este dice o piensa. La relación con el escenario no contribuye significativamente a la producción de sentido.

- Acontecimiento: la trama versa sobre un suceso que transcurre en el tiempo e involucra personajes.

- Abstracción: consiste en una composición de carácter conceptual que remite a una idea o un estado emocional. Puede contener personajes, objetos, símbolos.

\section{Contenidos}

Retomamos los tópicos propuestos por Wilson y Wilson (1987), que reagrupamos en categorías más abarcativas, a la vez que incluimos otras en función del corpus. Como se indicó anteriormente, las categorías se aplican de modo no excluyente. Algunas categorías tienen distinto grado de especificidad por lo que, para evitar posibles solapamien- 
tos, optamos por aquella más específica. Esto ocurrió con las categorías de juego y estados emocionales (véase abajo). Las categorías de contenidos son:

- Agresión: actos agresivos de diversa índole: burlas, actos delictivos, maltrato físico o intención de provocar la muerte (batallas, guerras). La violencia puede ser psicológica, física, institucional.

- Vida doméstica y pública: situaciones de la vida doméstica o familiar, creencias populares y celebraciones, tales como cumpleaños, inauguraciones, conciertos.

- Normas sociales: textos y manifestaciones de gran circulación social. Incluye normas sociales asociadas a aspectos morales, estereotipos, cuestiones relacionadas con la educación y política.

- Amor y amistad: avatares de los vínculos de pareja o amistad entre personajes.

- Juego: actividades lúdicas, eventos deportivos, actividades de tiempo libre y aventuras. Dado que estas actividades pueden ser parte de la vida cotidiana, cuando se codifica juego, no se codifica vida doméstica y pública.

- Equivocaciones o «meteduras de pata»: evento accidental, no intencional, que produce una reacción humorística en uno mismo o en otros.

- Tecnología: relaciones entre personajes e instrumentos cotidianos tecnológicos (celulares, computadoras, pantallas), así como maquinarias, transportes, entre otros.

- Naturaleza: relación del humano con elementos naturales (vegetales, animales) y con eventos climáticos.

- Estados emocionales: reacciones o estados emocionales que experimentan los personajes, tales como miedo, agobio, agotamiento, sorpresa, satisfacción. Los textos sobre relaciones de amor y amistad, en los cuales los personajes expresan afecto, no se codifican en esta categoría.

La codificación de los textos se realizó en tres pasos: 1) tres de las autoras codificaron independientemente los 151 textos humorísticos y luego se contrastaron dichas codificaciones. Las discrepancias fueron resueltas mediante discusión. 2) Un juez externo codificó el 15\% del corpus, lo que nos llevó principalmente a clarificar la descripción de algunas categorías y a incorporar otras categorías copresentes en el caso de la subdimensión contenidos. Se discutieron con este juez las codificaciones y se estableció un nuevo índice de acuerdo. 3) Otros cinco jueces externos codificaron las categorías excluyentes en $5 \%$ del corpus y se establecieron nuevos índices de acuerdo (tabla 1). 


\section{Tabla 1}

Indices de acuerdo en las dos instancias de codificación de jueces externos

\begin{tabular}{|c|c|c|c|}
\hline & Dimensiones & $\begin{array}{c}\text { Discusión } \\
\text { previa }\end{array}$ & $\begin{array}{l}\text { Discusión } \\
\text { posterior }\end{array}$ \\
\hline \multirow{5}{*}{$\begin{array}{l}\text { Juez experto } \\
\text { primera instancia }\end{array}$} & Motivación & $80 \%$ & $95 \%$ \\
\hline & Mundo-representación & $75 \%$ & $90 \%$ \\
\hline & Escala & $80 \%$ & $95 \%$ \\
\hline & Ontología & $85 \%$ & $90 \%$ \\
\hline & Contenidos & $95 \%$ & $100 \%$ \\
\hline \multirow{4}{*}{$\begin{array}{l}\text { Jueces expertos } \\
\text { segunda instancia }\end{array}$} & Motivación & $100 \%$ & $100 \%$ \\
\hline & Mundo-representación & $74 \%$ & $81 \%$ \\
\hline & Escala & $83 \%$ & $86 \%$ \\
\hline & Ontología & $83 \%$ & $83 \%$ \\
\hline
\end{tabular}

\section{Análisis estadísticos}

En primer lugar, se realizaron análisis descriptivos unidimensionales para apreciar la distribución global de los textos en las categorías de cada subdimensión. Luego, para el análisis articulado de los aspectos temáticos y pragmático-motivacionales, se aplicaron dos técnicas estadísticas de análisis multivariado: el análisis factorial de correspondencias múltiples (AFCM de ahora en adelante) y el análisis de clasificación jerárquica ascendente (ACJA; Greenacre, 1984), a través del software Spad 5.5.

El AFCM estudia un grupo de individuos (en este caso los 151 textos humorísticos) descriptos según variables nominales. El análisis permite establecer asociaciones entre las categorías de las variables y textos a partir de su proyección en planos factoriales. Las variables intervinientes pueden ser activas, cuando determinan la formación de los ejes factoriales, o ilustrativas, cuando se proyectan en los mismos sin incidir en su formación. A partir de las coordenadas obtenidas por los textos en los ejes del AFCM, el ACJA permite clasificarlos de forma exhaustiva y disjunta de acuerdo a cuán similares son entre sí, así como cuán diferentes son de otros textos: así, cada texto se asigna a una sola clase, junto a otros textos con los que comparte categorías distintivas. El índice de homogeneidad interna (IH) de cada clase es una medida de la similaridad entre los individuos que la componen (indicando o la mayor homogeneidad).

La dimensión motivación y las subdimensiones temáticas mundo-representación y escala se consideraron como variables activas. En cambio, las subdimensiones temáticas 
ontología y contenidos se consideraron ilustrativas. Debido a que las categorías de la dimensión contenidos se aplicaron de forma no excluyente, para integrarla en este análisis fue convertida en nueve variables dicotómicas: cada una expresa si la producción aborda o no el contenido en cuestión. La razón por la cual la dimensión ontología fue considerada ilustrativa fue su concentración en una sola de las categorías.

Por último, en los análisis multivariados también se consideraron como ilustrativas las siguientes variables:

- Formato de las producciones: viñeta única $(\mathrm{N}=77)$, tira $(\mathrm{N}=74)$.

- Rango etario de los participantes: distinguimos tres rangos en base a momentos del desarrollo y al número de participantes para lograr una división lo más balanceada posible: 10-12 años $(\mathrm{N}=36), 13-15$ años $(\mathrm{N}=40)$ y 16-19 años $\left(\mathrm{N}=5^{6}\right)$.

\section{Resultados}

En primer lugar, analizamos la distribución de la motivación de los autores y tres de las cuatro subdimensiones temáticas (figura 1).

Figura 1

Distribución de las categorías correspondientes a las subdimensiones

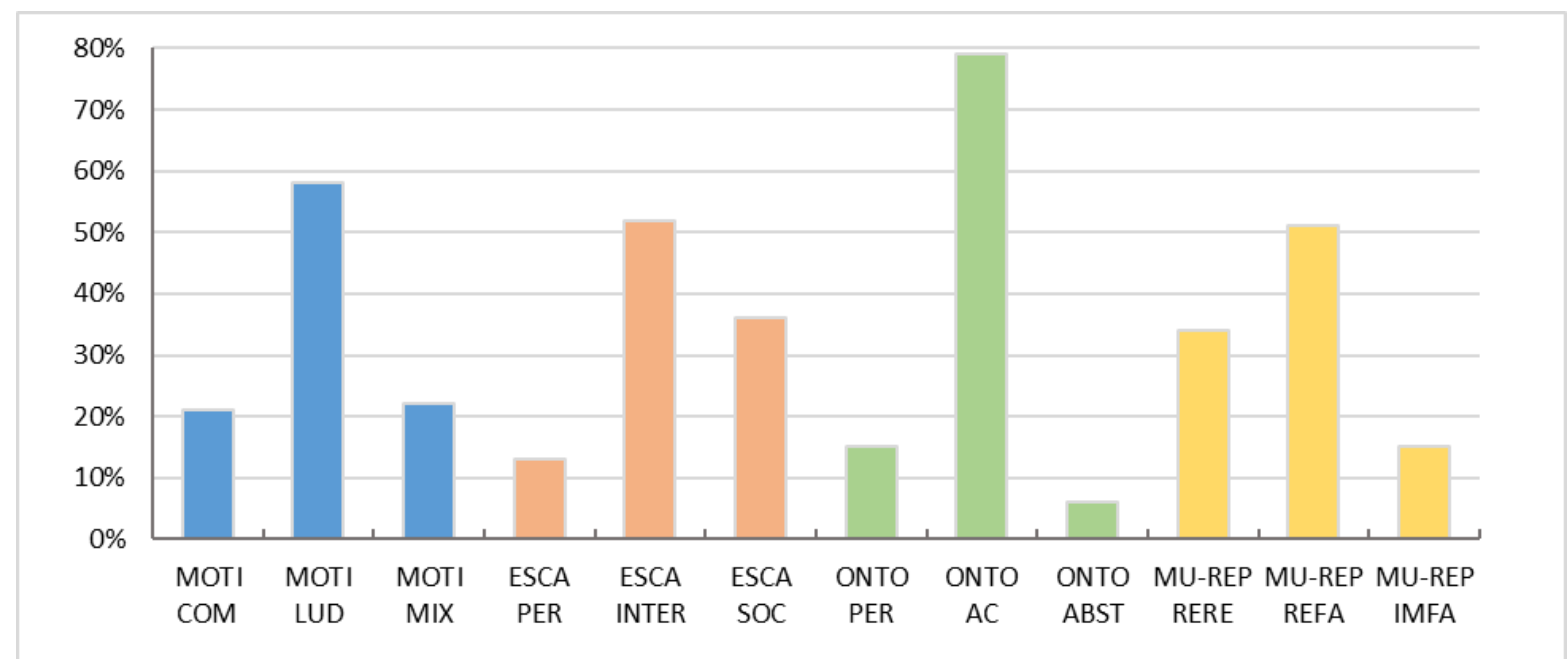

Nota. Subdimensiones motivación, escala, ontología y mundo-representación $(n=151)$. MOTI= motivación; COM: comprometida; LUD: lúdica; MIX: mixta. ESCA= Escala. PER: personal; INTER: interpersonal; SOC: social. ONTO= ontología. PER: personaje; AC: acontecimiento; ABST: abstracción. MU-REP= mundo-representación. RERE: real-realista; REFA: real-fantástico; IMFA: imaginario/mediático-fantástico. 
La preponderancia de la motivación lúdica está en consonancia con la concepción más generalizada del humor como fenómeno principalmente risible (figura 2). Las producciones comprometidas que no integran aspectos lúdicos constituyen un $20 \%$ (figura 3) y otro $22 \%$ aborda un tema de manera comprometida y a su vez lúdica (figura 4 ).

Figura 2

\section{Tania, 10 años}

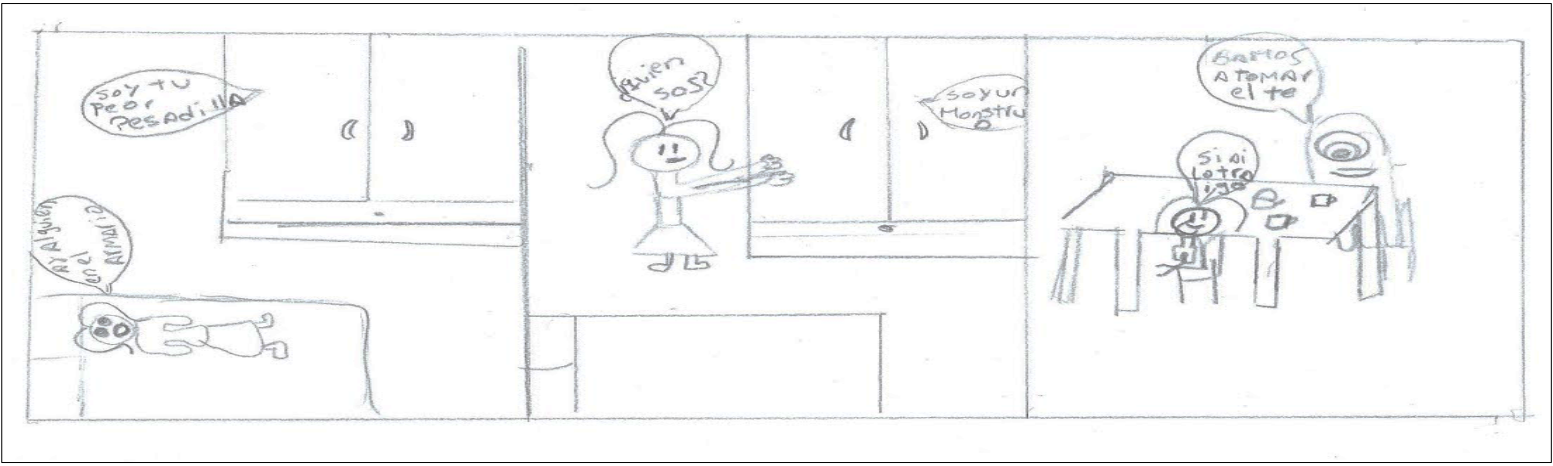

Nota. Viñeta 1. Niña: - Hay alguien en el armario. Voz: - Soy tu peor pesadilla. Viñeta 2. Niña: —¿Quién sos? Voz: - Soy un monstruo. Viñeta 3. Monstruo: —Vamos a tomar el té. Niña: —Sí, sí, ahí lo traigo.

Figura 3

Gala, 15 años

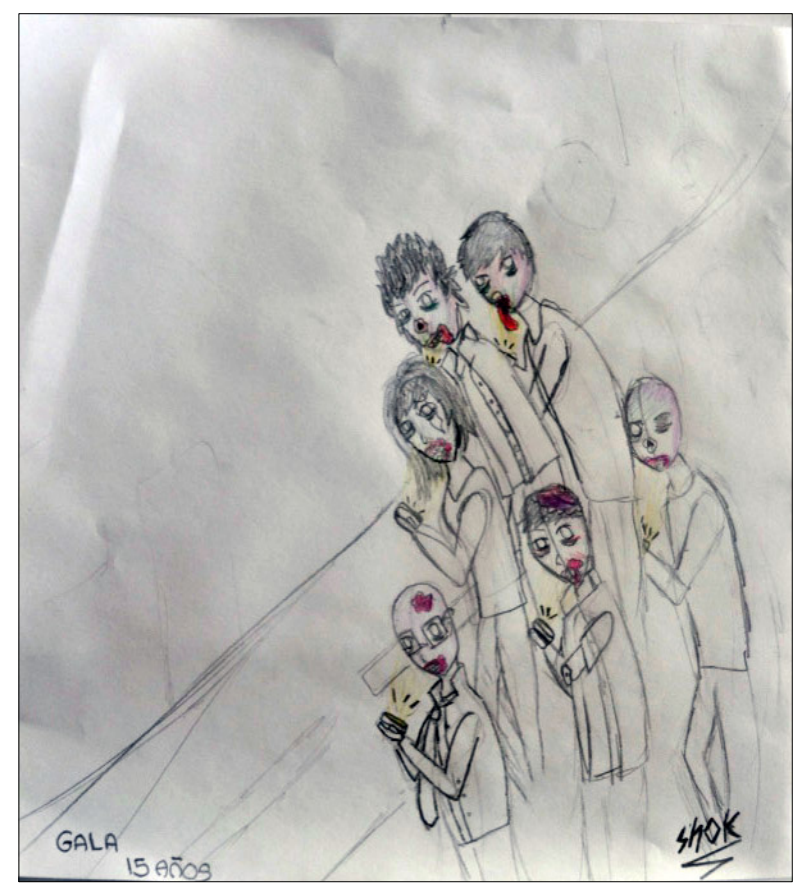


Figura 4

Santi, 17 años

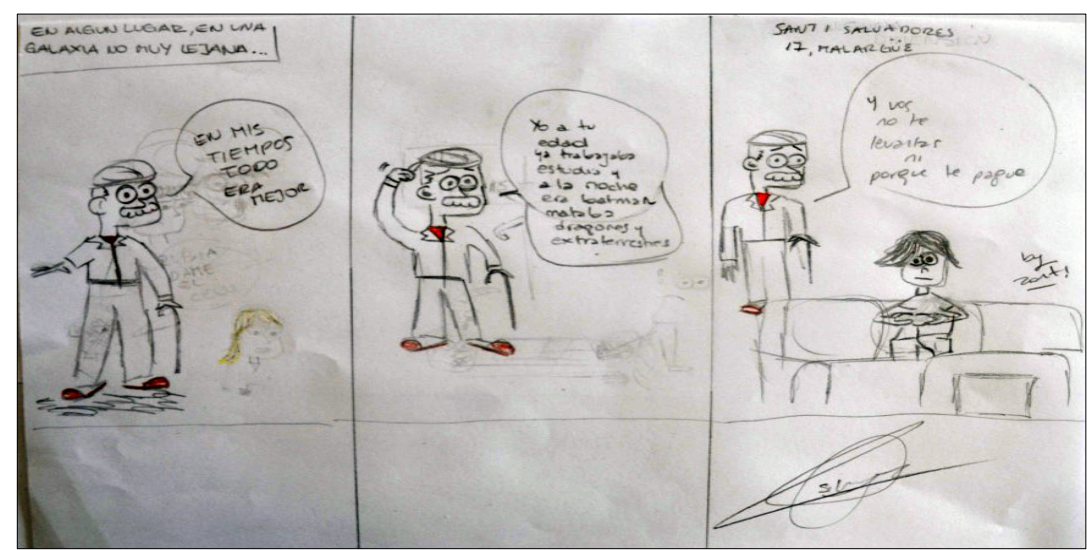

Nota. Viñeta 1. Cartucho: En algún lugar, en una galaxia no muy lejana... Abuelo: -En mis tiempos todo era mejor. Viñeta 2. Abuelo: - Yo a tu edad ya trabajaba y estudiaba y a la noche era Batman, mataba dragones y extraterrestres. Viñeta 3. Abuelo: $-\mathrm{Y}$ vos no te levantás ni porque te pague.

Más de la mitad de las producciones versan sobre temas cuya escala es interpersonal (figuras 2, 7 y 10), seguidas por la escala social (figuras 3, 4 y 6), ya sea en referencia a un grupo particular o a la humanidad en su totalidad. Solo un $13 \%$ remite a experiencias o reflexiones de la esfera interna de los personajes (figura 5). Hay una marcada preferencia por representar acontecimientos (figuras $2,3,4$ ), mientras que centrarse en un personaje constituye el $15 \%$ (figura 5 ). Las entidades abstractas que dan lugar a producciones conceptuales son excepcionales (figura 6).

\section{Figura 5}

Antonela, 12 años.

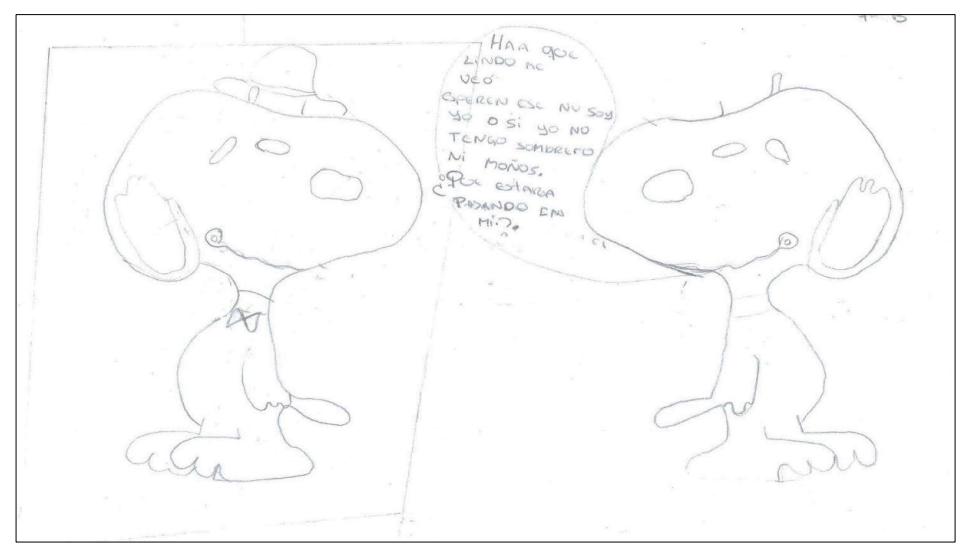

Nota. Snoopy: -Ahh, qué lindo me veo. Esperen, ese no soy yo, ¿ $\mathrm{O}$ sí? Yo no tengo sombrero ni moños. ¿Qué estará pasando en mí? 
Figura 6

Nicolás, 10 años

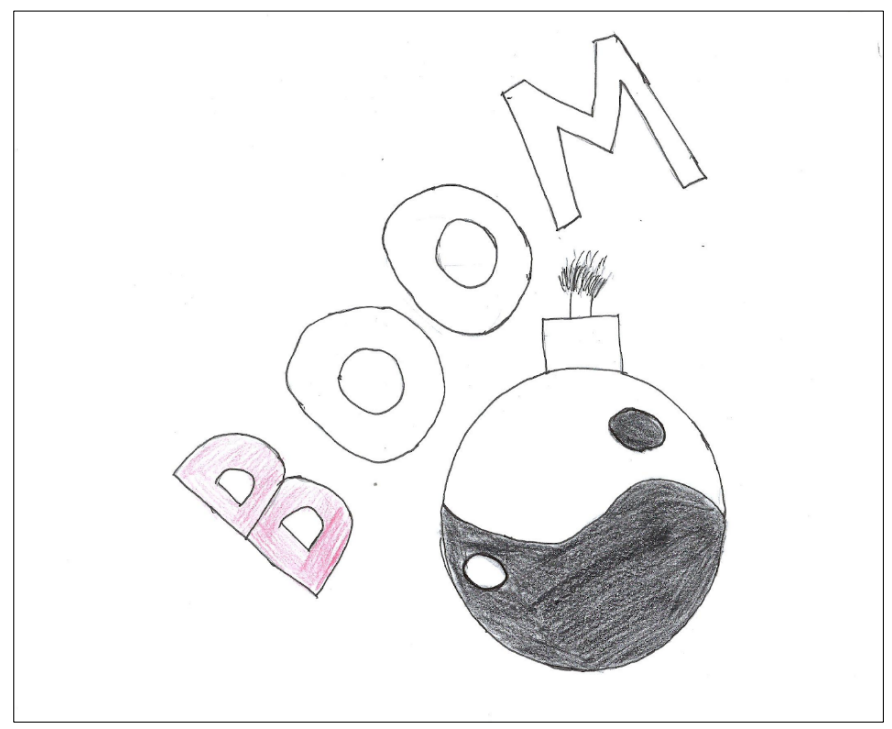

Por otra parte, la mayoría de las producciones aborda una situación del mundo real o posible, preferentemente a partir de personajes o situaciones imposibles o inverosímiles (figuras 2, 3 y 6). En menor medida, las temáticas realistas se representan de modo verosímil (figura 4 y 10) y solo pocas representan personajes o situaciones imaginarias, literarias o mediáticas, de una manera fantástica (figura 7 ).

Figura 7

Karen, 15 años

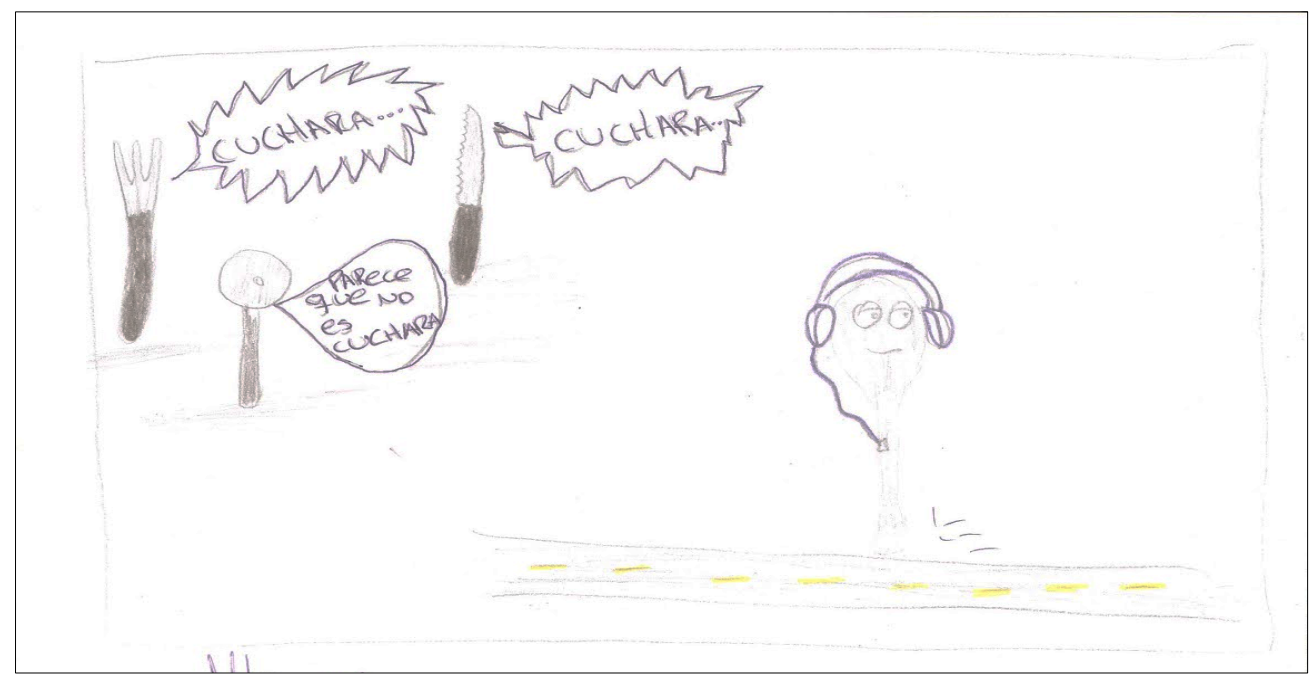

Nota. Tenedor: —Cuchara... Cuchillo: —Cuchara... Utensilio: —Parece que no escuchara. 
De los 151 textos, 79 presentaron más de un contenido (figura 8). En consonancia con su alta frecuencia, las coocurrencias más recurrentes asocian los contenidos agresivos y los contenidos sociales/normativos (por ejemplo, la figura 6). A su vez, todos los otros contenidos coocurren en mayor proporción con estas dos categorías, con excepción de los contenidos sobre vida doméstica y pública, que coocurren en su mayoría con los estados emocionales (ejemplos son las figuras 2 y 5 ) y la relación humano-tecnología (figuras 3 y 4 ).

Figura 8

Distribución de los contenidos

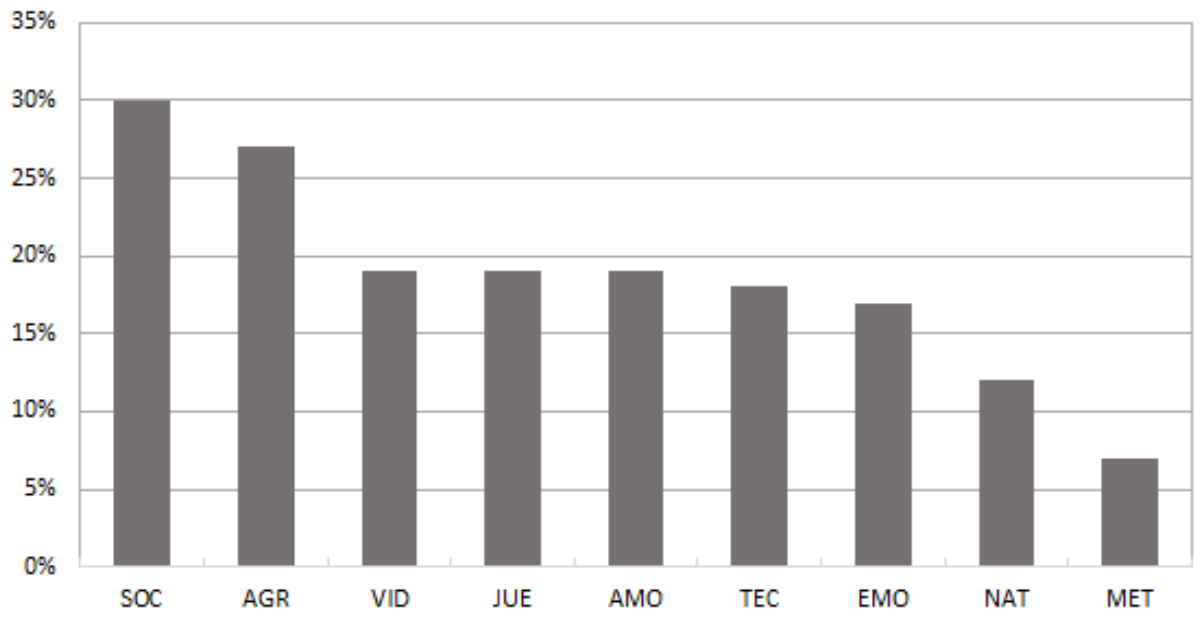

Nota. $(n=151)$. SOC: normas sociales. AGR: agresión. VID: vida doméstica y pública. JUE: juego. AMO: amor y amistad. TEC: tecnología. EMO: estados emocionales. NAT: naturaleza. MET: equivocaciones o «meteduras de pata».

Con el fin de caracterizar y distinguir los textos de acuerdo al conjunto de dimensiones motivacional y temáticas consideradas, realizamos un ACJA. Para ello consideramos los primeros cinco ejes factoriales del AFCM, que explican el $80 \%$ de la variabilidad de los datos. Seleccionamos la partición de seis clases que, a nuestro entender, es la que mejor describe la red de asociaciones entre la totalidad de las dimensiones y subdimensiones. Dos de ellas reúnen entre un quinto y un cuarto de los textos, mientras que las restantes algo más del 10\%. Las clases II y IV son las más homogéneas y la clase VI la más heterogénea. La figura 9 visualiza la ubicación de las seis clases en relación con las dimensiones y subdimensiones. 
Figura 9

Primer plano factorial: dimensiones, subdimensiones y clases

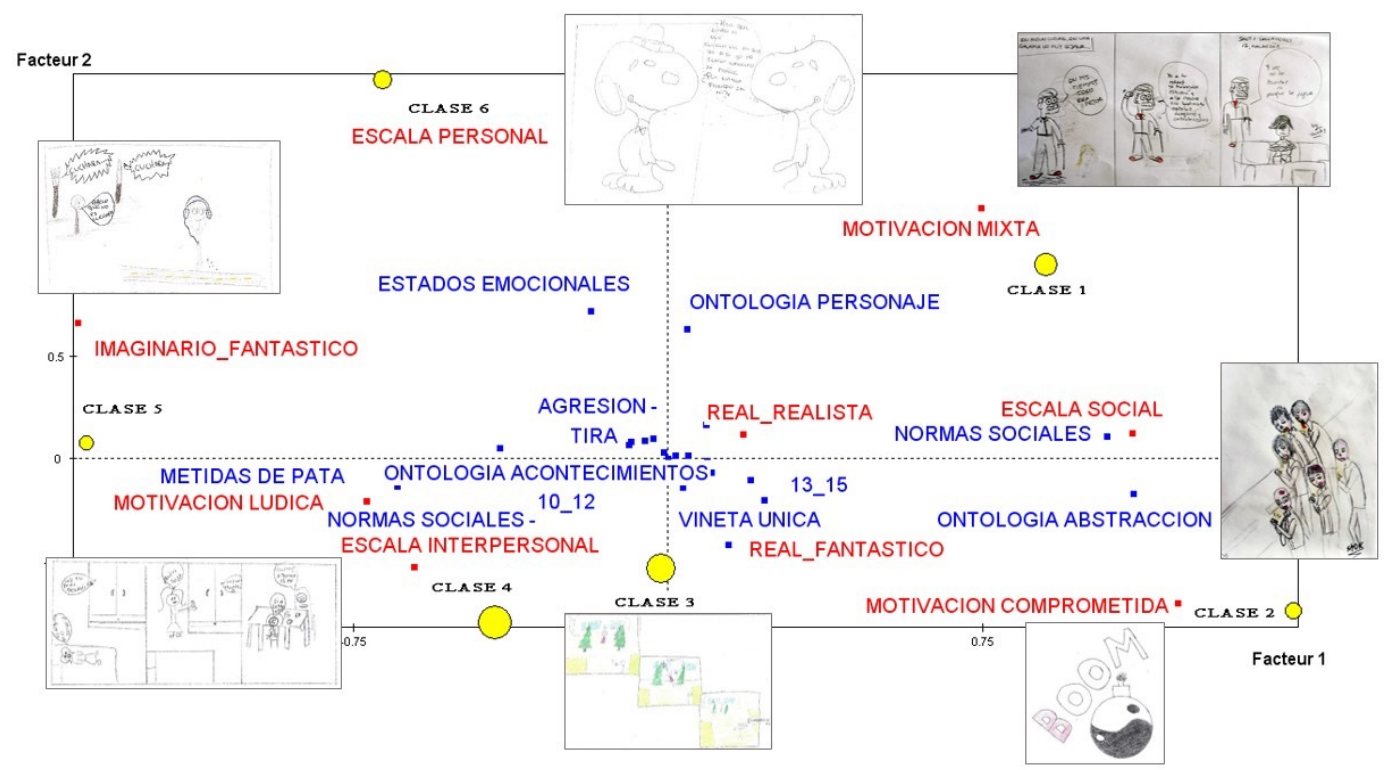

Nota. Categorías pragmático-motivacionales y temáticas que alcanzaron significación estadística: las seis clases de textos resultantes del análisis de clasificación jerárquica ascendente y los ejemplos de textos seleccionados para ilustrarlas.

\section{Clase 1: contenidos sociales con motivación mixta}

Reúne el $18 \%$ de los textos ( $\mathrm{IH}=0.104)$. Se caracteriza por una motivación mixta y trata temas a escala social. Si bien abundan las problemáticas sociopolíticas (estereotipos, educación, situaciones en las que se cuestionan o explicitan normas sociales), incluye otros temas de escala social como deportes, cuestiones generacionales, la relación de los humanos con la naturaleza. Un ejemplo es la figura 4, en la cual la crítica al desfase generacional y al lugar que ocupan los juegos electrónicos entre los jóvenes adquiere un tono divertido mediante la exageración y el absurdo.

Clase 2: viñetas únicas fantásticas sobre valores y normas sociales, con motivación comprometida

Reúne el $12 \%$ de los textos $(\mathrm{IH}=\mathrm{O})$. Se caracteriza por una motivación comprometida y por la escala social. Los temas son en su mayoría sobre normas sociales con énfasis en lo moral y se abordan preferentemente a partir de la construcción de una situación ficticia fantástica. La mayoría de estos textos se realiza en formato de viñeta única. Por ejemplo, en la figura 3 la autora critica y alerta sobre las consecuencias del uso de los celulares en las interacciones sociales. Esta clase incluye también los temas abordados me- 
diante abstracciones. En la figura 6, su autor buscó - tal como expresó en la entrevista-, representar a través del símbolo ying-yang la convivencia de las buenas y malas intenciones que subyacen en la realización de acciones.

\section{Clase 3: tiras realistas sobre acontecimientos interpersonales}

Reúne el $22 \%$ de los textos $(\mathrm{IH}=0.123)$. Estos remiten a acontecimientos reales o posibles, representados de forma realista y en formato de tira. Son características las metidas de pata o infortunios, entre otros temas diversos (actividades de la vida cotidiana, costumbres y creencias populares), que se dan en una escala interpersonal. La figura 1o representa una escena lúdica entre amigos, en la cual rompen un vidrio involuntariamente.

Figura 10

Luz, 15 años

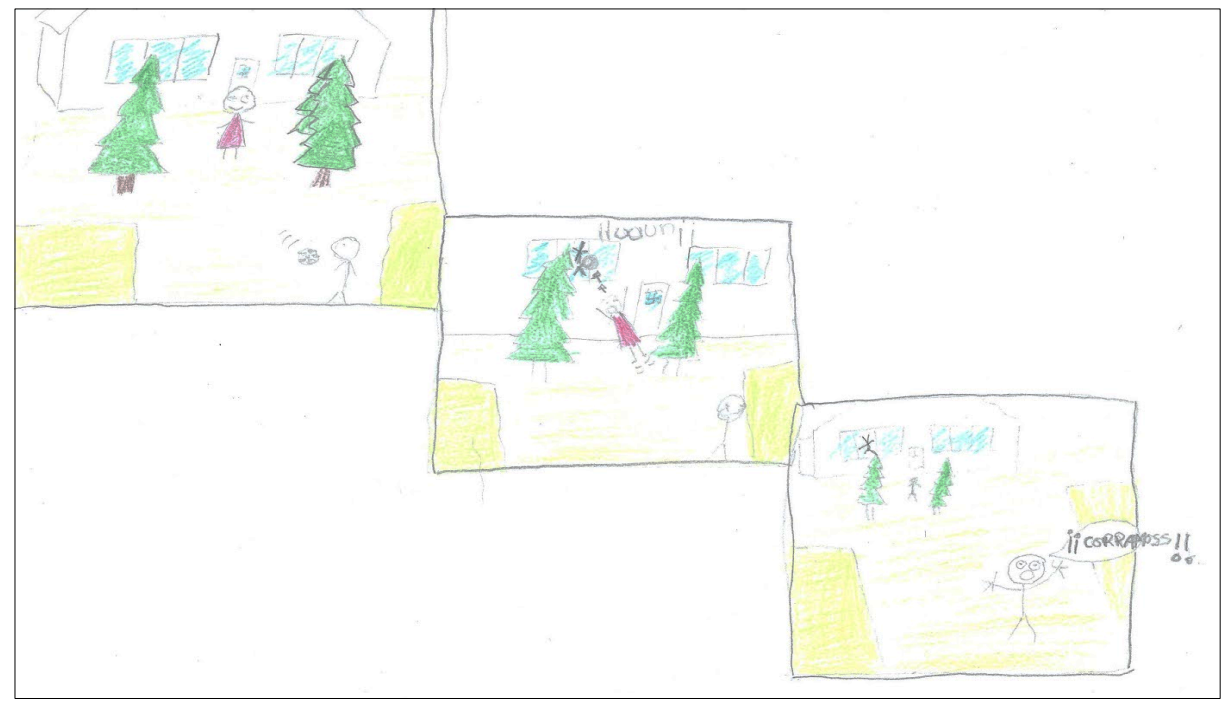

Nota. Cuadro 2. - Hoooo! Cuadro 3. -iiCorramos!!

\section{Clase 4: representación fantástica del mundo real a escala interpersonal con} motivación lúdica

Reúne el $25 \%$ de los textos $(\mathrm{IH}=0.035)$. Estos abordan un tema real o posible a escala interpersonal, a partir de elementos fantásticos desde una motivación lúdica. Los contenidos son variados y excluyen los de índole normativo o social. Son propios del grupo etario de 10 a 12 años. En la figura 2, una niña toma el té con un monstruo, dando lugar a una resolución inesperadamente amable a una situación que parecía terrorífica. 


\section{Clase 5: contenidos de alta circulación social con motivación lúdica}

Reúne el $11 \%$ de los textos $(\mathrm{IH}=0.026)$. Incluye textos lúdicos que versan sobre otros textos de alta circulación social, muchas veces a partir de la interacción entre personajes. La figura 7 recupera un chiste conocido propio del modo verbal oral, que juega con la personificación y el doble sentido.

\section{Clase 6: experiencias personales con expresión de estados emocionales}

Reúne el $12 \%$ de los textos ( $\mathrm{IH}=\mathrm{O} .152)$. Las viñetas focalizan en experiencias personales fundamentalmente de índole emocional. La figura 5 versa sobre el sentimiento de extrañeza del personaje al ver que el espejo no devuelve la imagen esperada, sugiriendo una reflexión de tipo existencial.

Con respecto a la variable etaria, solo la franja 10-12 caracteriza una de las clases. Complementariamente, los resultados obtenidos mediante el AFCM permitieron además identificar una asociación entre los participantes de 13-15 años y la motivación comprometida (un ejemplo es la figura 3).

\section{Discusión}

La revisión de estudios sobre los temas de producciones gráficas realizadas por niños puso de manifiesto la necesidad de desarrollar y sistematizar un conjunto comprehensivo y, a la vez, claramente delimitado de categorías. Ante este desafío, propusimos un abordaje multidimensional de aspectos temáticos, conjugando categorías generales con categorías más finas orientadas a un análisis en detalle. Así mismo, integramos la motivación del autor como un rasgo indispensable para estudiar la tarea creativa. El sistema desarrollado nos permitió así caracterizar nuestro corpus a nivel temático y motivacional. La consideración de un amplio rango etario permitió además detectar diferencias al crear humor gráfico que interpretamos en función de las distintas etapas del desarrollo de los participantes. Identificamos también variaciones en el formato de los textos según los temas y motivaciones elegidas.

Un primer resultado que es de interés retomar en esta sección, distingue los niños y las niñas entre 10 y 12 años de los adolescentes de 13 a 15 en función de sus motivaciones. Mientras los primeros crearon humor gráfico de carácter preferentemente lúdico, los segundos lo hicieron con compromiso. Este carácter lúdico identificado en los niños se asocia con producciones principalmente fantásticas, de escala interpersonal y con una 
subrepresentación de contenidos sociales/normativos. Contrariamente a lo esperado en función de la revisión bibliográfica, las equivocaciones o «meteduras de pata» no resultaron características de este grupo, sino que fueron compartidas por las tres franjas etarias. Sin embargo, mientras que los menores optaron por infortunios de carácter práctico (como ruptura de vidrios), muchas veces ligados a experiencias propias, los adolescentes basaron sus textos en observaciones de la vida cotidiana (por ejemplo, comportamientos de los gatos) y situaciones absurdas atemporales. Un corpus más amplio permitiría profundizar esta inicial observación.

Si bien los estudios sobre desarrollo nos llevaron a hipotetizar acerca de un interés por temas abstractos y sociopolíticos en la adolescencia tal como plantea Moshman (2009), encontramos una diversidad tanto motivacional como temática en la franja de 16 a 19 años. Esto daría cuenta de un amplio repertorio humorístico ligado a una concepción integral del humor como fenómeno risible o conmovedor en varios sentidos. Este resultado puede relacionarse con el trabajo de McGhee et al. (1990), quienes proponen que, a partir de la adolescencia tardía, el humor estaría más ligado a rasgos individuales relacionados con preferencias estilísticas que a cuestiones de desarrollo más generalizables.

Retomando el resultado que presentamos en el párrafo anterior, si analizamos en detalle un contenido específico frecuentemente abordado en estudios de humor y desarrollo - como es la burla-, sí observamos una preponderancia de la escala social en los adolescentes, en línea con Bariaud (2013). En efecto, la burla toma la forma de una crítica social por parte del autor, contrapuesto a lo que ocurre en la franja de 10 a 12 años, en la cual son los personajes los que se burlan entre sí por diferentes aspectos o situaciones (equivocaciones, apariencia física, cuestiones escatológicas). En un próximo estudio nos interesa focalizar en aquellas producciones que se enmarcan dentro de lo que hemos denominado «humor serio» (Flores, 200o) y que tiene a la crítica social como principal finalidad. En este sentido, cobra particular interés caracterizar contenidos y retórica de dichas producciones e indagar acerca del carácter satírico de las mismas.

Cabe mencionar dos características ligadas a cuestiones del género discursivo. La primera refiere a la preponderancia del tratamiento de situaciones reales o posibles a partir de representaciones fantásticas, tal como ocurre en el humor gráfico que circula en la prensa. En efecto, los autores gozan de gran libertad para construir las situaciones ficticias en las que se juega con sentidos propios y figurados, empleando recursos como la personificación, la metáfora y la hipérbole. El establecimiento de una incongruencia humorística es propiciada por la ruptura que este juego de sentidos habilita, subvirtiendo 
la dupla real-realista. La segunda característica remite al formato de los textos. Encontramos que la viñeta única predomina cuando los autores abordan temas sociales del mundo real de forma comprometida a partir de la construcción de una situación fantástica. Este formato también fue el elegido para las composiciones abstractas. Si conectamos estos hallazgos con un estudio previo (Pedrazzini et al., en prensa), en el que la viñeta única se asoció con incongruencias conceptuales, podríamos hipotetizar sobre un mayor nivel de complejidad cognitivo exigido en este formato. Interpretamos esta red de asociaciones por el carácter sintético de la viñeta única, la cual le permite al autor lograr un mensaje eficaz en su objetivo de interpelar e impactar al lector. La tira, en cambio, fue la predilecta para desplegar acontecimientos reales o posibles donde están involucrados diferentes personajes, como en las metidas de pata. Este hallazgo está en sintonía con lo propuesto por Puche-Navarro y Lozano-Hormaza (2002) y remite a la temporalidad característica del formato.

Con respecto a la posible incidencia de las condiciones sociales de producción en el tratamiento de algunos contenidos en los textos - en línea con lo planteado por Einarsdottir et al. (2009) -, observamos que, si bien los contenidos escatológicos son habituales en el repertorio humorístico desde temprana edad y hasta la infancia tardía, son muy poco frecuentes en nuestro corpus. Esto podría explicarse por la presencia de pares, docentes e investigadoras en el taller, así como por la entrevista final. En este sentido, la posible autocensura en la selección temática de las producciones realizadas podría considerarse como una limitación del estudio.

Este trabajo se enmarca dentro de un proyecto más general orientado a poner de relieve el potencial educativo que reviste la producción de humor gráfico, en tanto actividad altamente agentiva en la cual los participantes se enfrentan a la posibilidad de tomar perspectiva respecto de una temática que los convoca y que pueden expresar libre y multimodalmente, comprometiendo procesos motivacionales, imaginativos y expresivos. Las posibles aplicaciones en el ámbito educativo son muy diversas. Si bien el humor gráfico puede utilizarse como disparador en el abordaje de otros temas curriculares y comunitarios, reivindicamos su aprovechamiento como medio de comunicación en sí mismo. La creación de humor gráfico exige y promueve un cierto dominio de los modos verbal escrito y visual, así como la capacidad de articularlos de forma eficaz e inteligible. En este sentido, es un recurso especialmente rico en los procesos de alfabetización multimodal (Teubal \& Guberman, 2014). 
Por último, consideramos relevante destacar algunas características del diseño e implementación de los talleres realizados. En el taller exploratorio - que no contó con introducción previa-, los participantes resolvieron la consigna manteniéndose en el terreno de lo convencional mediante la representación mayoritaria de chistes conocidos. Esto contrastó significativamente con lo sucedido en los talleres diseñados para este estudio tal y como se han descrito. En ellos observamos y evaluamos que la propuesta de una presentación variada en un clima participativo y distendido dio lugar a que los participantes se animaran a explorar temas, recursos y otros tipos de humor, más allá del exclusivamente lúdico. Teniendo en cuenta los beneficios de un trabajo sostenido como puede apreciarse en muchas experiencias realizadas en otros campos de la alfabetización, es de esperar que la implementación de un taller de producción e interpretación de humor gráfico de larga duración promueva un aprendizaje más profundo de este medio, así como del dibujo y su articulación con la escritura como modos de comunicación. Futuros trabajos focalizarán en el proceso de aprendizaje de este género a partir de un taller trimestral. Con este foco educativo, una futura línea de investigación podría profundizar en el estudio de estrategias pedagógicas que favorezcan el uso reflexivo considerando el potencial de cada modo semiótico, no solo para expresarse y comunicar, sino también para pensar y resignificar el mundo (véanse los trabajos de Papandreou, 2014 y Pantaleo, 2014, quienes señalan estas cuestiones como parte de la agenda investigativa pendiente).

\section{Agradecimientos}

Agradecemos a las niñas, niños y adolescentes participantes de los talleres, a los docentes y directivos involucrados; a Montserrat de la Cruz y a los miembros del grupo interdisciplinario en Desarrollo Cognitivo, Aprendizaje y Comunicación por colaborar en el proceso de control interjuez.

\section{Referencias}

Apter, M. (1991). A structural-phenomenology of play. En J. Kerr \& M. Apter (Eds.), Adult play: A reversal theory approach (13-29). Swets \& Zeitlinger.

Attardo, S. (1997). The semantic foundations of cognitive theories of humor. Humor: International Journal of Humor Research, 10(4).395-420. https://doi.org/10.1515/ humr.1997.10.4.395 
Attardo, S., \& Raskin, V. (1991). Script theory revis(it)ed: Joke similarity and joke representation model. Humor: International Journal of Humor Research, 4(3-4). 293-347. https://doi.org/10.1515/humr.1991.4.3-4.293

Bariaud, F. (2013). Age differences in children's humor. En P. McGhee (Ed.), Humor and children's development: A guide to practical applications (21-42). Routledge.

Bombi, A. \& Pinto, G. (1993). Los colores de la amistad: estudios sobre las representaciones pictóricas de la amistad entre los niños. Visor.

Bugallo, L., Zinkgräf, C., \& Pedrazzini, A. (2018a). El humor gráfico: una herramienta potente para enseñar y aprender. Contextos de Educación, (24), 55-65.

Bugallo, L., Zinkgräf, C., \& Pedrazzini, A. (2018b, abril). Juego con signos y sentidos: el humor gráfico como campo de desafío para niños y adolescentes [Ponencia]. VII Congreso Nacional y V Internacional de Investigación Educativa «Políticas y prácticas de producción y circulación de conocimiento: a 20 años del I Congreso de Investigación Educativa en la UNCo». Cipolletti, Argentina.

Burkitt, E., Watling, D., \& Murray, L. (2011). Children's drawings of significant figures for a peer or an adult audience. Infant and Child Development, 2o(6), 466-473. https://doi.org/10.1002/icd.735

Charaudeau, P. (2006). De nouvelles catégories pour l'humour? Questions de communication, (10), 19-41. https://doi.org/10.400o/questionsdecommunication.7688

Chi, M. (1992). Conceptual change within and across ontological categories: Examples for learning and discovery in science. En R. Giere (Ed.), Cognitive models of science: Minnesota studies in the philosophy of science (pp. 129-186). University of Minnesota Press.

Chi, M. (2008). Three types of conceptual change: Belief revision, mental model transformation, and categorical shift. En S. Vosniadou (Ed.), Handbook of research on conceptual change (pp. 61-82). Lawrence Erlbaum.

Einarsdottir, J., Dockett, S., \& Perry, B. (2009). Making meaning: Children's perspectives expressed through drawings. Early Child Development and Care, 179(2), 217-232. https://doi.org/10.1080/03004430802666999

Faulkner, D., \& Coates, E. (2011). Exploring children's creative narratives. Routledge. Flores, A. (2000). Humor y posmodernidad: el humor serio. Ferreyra editor.

Freeman, N. (1980). Strategies of representation in young children: Analysis of spatial skills and drawing processes. Academic Press.

Freud, S. (1992). El humor. Amorrortu. (Obra original publicada en 1927).

Führ, M. (2002). Coping humor in early adolescence. Humor: International Journal of Humor Research, 15(3), 283-304. https://doi.org/10.1515/humr.2002.016 
Glaser, B., \& Strauss, A. (1967). The discovery of grounded theory: Strategies for qualitative research. Aldine Publishing Company.

Greenacre, M. J. (1984). Theory and applications of correspondence analysis. Academic Press.

Hess-Zimmermann, K. (2014). Desarrollo del humor en los años escolares: la reflexión metalingüistica de chistes referenciales. Estudios de Lingüística Aplicada, 32(60), 57-87.

Karakos, H. (2015). Understanding civic engagement among youth in diverse contexts. Vanderbilt University.

Klein, D., \& Kuiper, N. (2006). Humor styles, peer relationships, and bullying in middle childhood. Humor: International Journal of Humor Research, 19(4), 383-404.

https://doi.org/10.1515/HUMOR.2006.019

Kress, G. (2010). Multimodality: A social semiotic approach to contemporary communication. Routledge.

Labitsi, V. (2008). Spontaneous narrative drawing at the age of six: Pantelis case. Revista da Associação de Professores de Expressão e Comunicação Visual, (50), 7-11.

Levorato, Ch., \& Cacciari, C. (2002). The creation of new figurative expressions: Psycholinguistic evidence in Italian children, adolescents and adults. Journal of Child Language, 29(1), 127-150. https://doi.org/10.1017/S0305000901004950

Martin, R. A., \& Ford, T. E. (2018). The psychology of humor: An integrative approach. Elsevier.

McCloud, S. (1994). Understanding comics: The invisible art. Harper Perennial.

McGhee, P. (1979). Humor: Its origin and development. Freeman.

McGhee, P. (2002). Understanding and promoting the development of children's humor. Kendall/Hunt.

McGhee, P., Ruch, W., \& Hehl, F.-J. (1990). A personality-based model of humor development during adulthood. Humor: International Journal of Humor Research, $3(2)$, 119-146. https://doi.org/10.1515/humr.1990.3.2.119

Milreault, G., \& Reddy, V. (2016). Humor in infants: Developmental and psychological perspectives. Springer International Publishing.

Moshman, D. (2009). Adolescence. En U. Müller, J. Carpendale, \& L. Smith (Eds.), The Cambridge Companion to Piaget (255-269). Cambridge University Press. https:// doi.org/10.1017/CCOL9780521898584.011

Ospina-Ramírez, D. A., López-González, S., Burgos-Laitón, S. B., \& Madera-Ruiz, J. A. (2018). La paz entre lo urbano y lo rural: imaginarios de paz de niños y niñas sobre el posconflicto en Colombia. Revista Latinoamericana de Ciencias Sociales, Niñez y Juventud, 16(2), 943-960. https://doi.org/10.1160o/1692715x.16220 
Pedrazzini, A., Bugallo, L., Zinkgräf, C., \& Scheuer, N. (en prensa). Adolescents creating cartoons: A developmental study of humor. Humor: International Journal of Humor Research.

Pedrazzini, A., \& Scheuer, N. (2010). La interacción lingüística e ícono-plástica en la producción de caricaturas políticas: un estudio funcional y retórico. Irice. Nueva Época, (21), 95-111.

Pedrazzini, A., \& Scheuer, N. (2018). Distinguishing cartoon subgenres based on a multicultural contemporary corpus. European Journal of Humour Research, 6(1), 100-123. https://doi.org/10.7592/ejhr2018.6.1.pedrazzini

Piret, R. (1940). La genèse du sens du comique chez l'enfant. University of Liège.

Pitri, E. (2011). Children's funny art and the form it can take over time. International Journal of Education through Art, 7(1), 81-96. https://doi.org/10.1386/eta.7.1.81_1

Puche-Navarro, R. \& Lozano-Hormaza, H. (2002). El sentido del humor en el niño. Siglo del Hombre Editores; Universidad del Valle.

Rew, L., Tyler, D., Fredland, N., \& Hannah, D. (2012). Adolescents' concerns as they transition through high school. Advances in Nursing Science, 35(3), 205-221. https://doi.org/10.1097/ans.obo13e318261a7d7

Steimberg, O. (2013). Leyendo historietas: textos sobre relatos visuales y humor gráfico. Eterna Cadencia Editora.

Teubal, E., \& Guberman, A. (2014). Graphic texts: Literacy enhancing tools in early childhood. Sense. https://doi.org/10.1007/978-94-6209-710-0

Vilches, L. (1984). La lectura de la imagen: prensa, cine, televisión. Paidós.

Wilson, B., \& Wilson, M. (1987). Pictorial composition and narrative structure: Themes and the creation of meaning in the drawings of egyptian and japanese children. Visual Arts Research, 13(26), 10-21.

Wright, S. (2007). Graphic-narrative play: Young children's authoring through drawing and telling. International Journal of Education \& the Arts, 8(8), 1-11. 